\title{
Energy Efficiency Analysis and Design Optimization of an Actuation System in a Soft Modular Lower Limb Exoskeleton
}

\author{
Jesús Ortiz $^{1}$, Tommaso Poliero ${ }^{1}$, Giovanni Cairoli ${ }^{2}$, Eveline Graf ${ }^{3}$ and Darwin G. Caldwell ${ }^{1}$
}

\begin{abstract}
One of the critical aspects in the design of an assistive wearable robot is the energy efficiency of the actuation system, since it affects significantly the weight and consequently the comfort of the system. Several strategies have been used in previous research, mostly based on energy harvesting, compliant elements for mechanical energy accumulation (springs or elastic cords), ratchets and clutches. However, the design of the optimal actuator arrangement is highly dependent on the task, which increases significantly the complexity of the design process.

In this work we present an energy efficiency analysis and design optimization of an actuation system applied to a soft module lower limb exoskeleton. Instead of performing a comparison between predefined mechanism arrangements, we solve a full optimization problem which includes not only the mechanism parameters, but also the mechanism architecture itself. The optimization is performed for a walking task using gait data from a stroke subject, and the result is a set of actuator arrangements with optimal parameters for the analyzed task and selected user. The optimized mechanism is able to reduce the energy requirements by $20-65 \%$, depending of the joint. The proposed mechanism is currently under development within the XoSoft EU project, a modular soft lower-limb exoskeleton to assist people with mobility impairments.
\end{abstract}

Index Terms-Prosthetics and Exoskeletons; Wearable Robots; Mechanism Design.

\section{INTRODUCTION}

$\mathbf{I}$ $\mathrm{N}$ recent years, there have been very important developments in the area of wearable assistive robots [1]. Most common applications are in the rehabilitation area [2], but there are other fields using exoskeletons, such as military [3], [4], or industrial applications [5]. The comfort of the system is one of the key aspects, independently of the application, being the weight one of the major sources of discomfort. The autonomy of the system, a common requirement of a wearable device, affects negatively the weight of the system. Longer autonomy requirements imply bigger and heavier battery packs.

Manuscript received: May, 24th, 2017; Revised September, 11th, 2017; Accepted October, 9th, 2017.

This paper was recommended for publication by Editor Allison M. Okamura upon evaluation of the Associate Editor and Reviewers' comments. This work has received funding from the European Union's Horizon 2020 framework programme for research and innovation under grant agreement No 688175 .

${ }^{1}$ Jesús Ortiz, Tommaso Poliero and Darwin G. Caldwell are with the Department of Advanced Robotics, Istituto Italiano di Tecnologia, via Morego, 30, 16163 Genova \{jesus.ortiz, tommaso.poliero, darwin.caldwell\}eit.it

${ }^{2}$ Giovanni Cairoli is with the Design Factors Research Group, University of Limerick, Limerick, Ireland cairoli.giovanni@gmail.com

${ }^{3}$ Eveline Graf is with the Institute of Physiotherapy, ZHAW Zurich University of Applied Sciences, Winterthur, Switzerland gravezhaw.ch

Digital Object Identifier (DOI): see top of this page.
For that reason the simplicity and energy efficiency of the actuation system plays a very important role in the design of a wearable assistive device.

Recent developments in the area of assistive wearable devices are making use of soft technologies instead of rigid structures [6]. The use of this kind of technologies, improves significantly the comfort of the system, but the weight requirements are even more critical, above all in the case of assistance of people with mobility impairments. Recent studies show that the wearability of such systems is one of the critical aspects [7] for this kind of users.

In order to reduce the requirements of the actuation system, in terms of torque and power, it is possible to use passive or quasi-passive elements combined with classical actuators. This solution has been explored previously by [8], where the authors propose a quasi-passive mechanism for the lower limbs based on springs, clutches and variable damping mechanisms. A simplification of this concept was successfully implemented by [9] on a hard exoskeleton. Also the Maccepa mechanism [10], [11], [12] has been demonstrated to be highly efficient in the use of wearable robots. Other combination of compliant elements can be found in literature, such as [13], [14], [15], [16] and more recently [17] on a soft elbow exoskeleton. The use of compliant elements in combination with an actuation system can be also found in other robotic areas. A good example is [18] where the authors use an asymmetric compliant actuator scheme using elastic cords instead of metal springs, reducing considerably the energy requirements.

Most of the previous work proposing such mechanisms, perform some kind of estimation or optimization of the system parameters [8], [9]. That is, given an actuator system arrangement, find the best parameters which minimizes the energy or any other cost function. Alternatively, some works try to optimize the geometry and topology of the compliant elements ([19], [20] and [21]). However, it is still necessary to start from a basic mechanical design.

In this paper, we present an analysis and design optimization of an actuation system in a soft lower limb wearable assistive device. Since each pathology and subject are expected to have different requirements, we focus this work on a single subject to design ad-hoc devices. Section II describes a simplified formulation for the design optimization of a mechanism, including not only the optimization of the different parameters of the system, but also the element arrangement itself. Using this method, we optimize mechanisms for the ankle, knee and hip joints using real gait data from a stroke subject. 
The gait data used for the design is presented in section III, while the optimization and design of the proposed mechanism is described in section IV. We conclude the paper with the conclusions and future work in section $\mathrm{V}$.

\section{FORMULATION}

In order to elaborate the formulation for the description of the actuation system, we need to take into consideration the scope of this paper, which is the design of a mechanism to assist a specific movement and subject. In other words, we do not want an actuation system to generate a trajectory, but to reduce the force and power required by the person to generate the motion. Conceptually, if $F_{b i o}$ is the force applied by the person and $F_{\text {exo }}$ is the force applied by the exoskeleton mechanism, the new required force can be expressed in the following way:

$$
F_{r e q}=F_{b i o}-F_{\text {exo }}
$$

In the ideal case where the exoskeleton is able to generate all the force to generate the movement, the new required force will be zero:

$$
F_{\text {exo }} \rightarrow F_{b i o} \Rightarrow F_{r e q} \rightarrow 0
$$

In the remainder of this section, we give a detailed description of the formulation used to simulate an actuation system composed by a number of mechanical elements such as motors, springs and dampers. At the end of this section, we arrive to the simplified formulation which allows the simulation and optimization of an arbitrary actuator arrangement.

\section{A. Actuator equation}

The total force applied by an actuator $F$ can be expressed according to the following equation:

$$
F=A(\chi)+K(\chi) \cdot x+C(\chi) \cdot \dot{x}+M(\chi) \cdot \ddot{x}
$$

where $\chi$ is the system status, which includes time, position, velocity, acceleration, and any other input:

$$
\chi=(t, x, \dot{x}, \ddot{x}, \ldots)
$$

and $A(\chi)$ is the controlled force of the actuator; $K(\chi)$ is the force generated by any elastic term of the system; $C(\chi)$ is the force generated by any damping term of the system; and $M(\chi)$ is the mass/inertia term. The terms $A, K, C$ and $M$ depend on the system status and can be controlled.

The equation 3 is generic and can represent also non actuated elements such as springs, dampers and clutches. In Table I we can see an example of how these terms would be for some ideal elements.
TABLE I

IDEAL ELEMENTS

\begin{tabular}{l|cccc}
\hline Element & $A(\chi)$ & $K(\chi)$ & $C(\chi)$ & $M(\chi)$ \\
\hline Spring & $k \cdot x_{0}$ & $-k$ & 0 & 0 \\
Damper & 0 & 0 & $-c$ & 0 \\
Clutch & 0 & 0 & $-\infty / 0$ & 0 \\
\hline
\end{tabular}

\section{B. Discretization}

The equation 3 is an non-homogeneous Ordinary Differential Equation (ODE) which can be solved numerically using different methods. We apply a first approximation of the solution considering the coefficients quasi-constant. This simplifies the problem, since we can consider our system as a linear ODE. Using a second order time stepping method, we can express the equation 3 in the following discretized way:

$$
F(t+h)=\alpha(\chi)+\beta(\chi) \cdot \ddot{x}(t)
$$

where $t$ is the current time, $h$ is the time step,

$$
\begin{aligned}
\alpha(\chi)= & A(\chi)+ \\
& K(\chi) \cdot[x(t)+\dot{x}(t) \cdot h]+ \\
& C(\chi) \cdot \dot{x}(t)
\end{aligned}
$$

and

$$
\beta(\chi)=M(\chi)+C(\chi) \cdot h+K(\chi) \cdot \frac{1}{2} \cdot h^{2}
$$

\section{Element combination}

Using the previous formulation, it is easy to combine different elements in series or in parallel. In case of elements in parallel, the total force would be the sum of the force of each singular element, leading to the following equation:

$$
\begin{aligned}
F & =\sum_{e=1}^{N}\left[\alpha_{e}(\chi)+\beta_{e}(\chi) \cdot \ddot{x}\right] \\
& =\sum_{e=1}^{N} \alpha_{e}(\chi)+\sum_{e=1}^{N} \beta_{e}(\chi) \cdot \ddot{x} \\
& =\alpha_{p}(\chi)+\beta_{p}(\chi) \cdot \ddot{x}
\end{aligned}
$$

We can do a similar analysis for elements in series. In this case, the force of each element would be the same, but the total displacement would be the sum of all the elements. This leads to the following equation:

$$
\begin{aligned}
F & =\frac{\sum_{e=1}^{N} \frac{\alpha_{e}(\chi)}{\beta_{e}(\chi)}}{\sum_{e=1}^{N} \frac{1}{\beta_{e}(\chi)}}+\frac{1}{\sum_{e=1}^{N} \frac{1}{\beta_{e}(\chi)}} \cdot \ddot{x} \\
& =\alpha_{s}(\chi)+\beta_{s}(\chi) \cdot \ddot{x}
\end{aligned}
$$

As we can see, the equations 8 and 9 have the same form as the equation 5 , which means that we can use the same solution system for one element, or for a group of elements in parallel or series. 


\section{Optimization}

As explained at the beginning of this section, the target is to select the actuator arrangement and its parameters which approximates better the biological forces. This problem can be solved using classical continuous optimization algorithms. From a practical point of view, we selected the residual energy as the cost function instead of the force, as stated in the equation 10 .

$$
E(\xi)=h \cdot \sum_{t=0}^{T}\left|F(t) \cdot \dot{x}(t)-P_{b i o}(t)\right|
$$

where $\xi$ is the set of parameters, $T$ is the total time of the motion and $P_{b i o}(t)$ is the power reference.

It is also possible to use a different cost function to take into account other aspects such as energy consumption or weight. For example a clutch would consume more energy than a passive spring, or a complex arrangement of elements would weight more than a single passive element.

Note that in our case we do not want to minimize the error (the difference between the force generated by the mechanism $F_{\text {exo }}$ and the biological force $F_{b i o}$ ), but the residual energy required to generate the target motion. The energy is the objective function of our optimization problem, which depends on the parameters of the system. Each parameter can be bounded, giving the following set of inequality constrains:

$$
\xi_{i}^{\min } \leq \xi_{i} \leq \xi_{i}^{\max }
$$

where $\xi_{i} \in \xi$, and $\xi_{i}^{\min }$ and $\xi_{i}^{\max }$ are the boundings of the parameters.

We used the Nelder-Mead method [22] to solve the optimization problem. However, the convergence of this method depends on the starting point, since it could find a local minima. In our case, this aspect can be a problem, since our final target is to find the best mechanism and not simply the best parameters. For this reason we do not know a priori the mechanism architecture and we cannot choose empirically the initial parameter set. To increase the robustness of the Nelder-Mead method we perform a random sampling within all the parameter space, and we use the parameter sets with the smallest errors to set the vertices of the initial simplex.

\section{GAIT DATA}

In order to optimize a mechanism arrangement using the method explained in the previous section, we need some target kinematic and dynamic data. In particular, in this section we present the gait data of a 68 years old post stroke subject with unilateral gait impairment (see Table II). Three or four retroreflective markers were attached to different body segments (forefoot, rearfoot, shank, thigh, pelvis and trunk) and recorded by a 3D-highspeed camera system with 12 cameras (Vicon Vantage V5, Vicon Motion Systems Ltd, Oxford, UK) with a sampling rate of $240 \mathrm{~Hz}$. Simultaneously, two AMTI force plates (OR6-7-2000, AMTI Inc, Watertown, USA) recorded ground reaction forces at $1200 \mathrm{~Hz}$. Before analysis, data was filtered using a low-pass Butterworth filter (4th-order) with a cut-off frequency of $7 \mathrm{~Hz}$ (kinematic) and $400 \mathrm{~Hz}$ (kinetics).
TABLE II

SUBJECT AND GAIT DATA INFORMATION

\begin{tabular}{l|c}
\hline Gender & male \\
Age & 68 years \\
Height & $170.0 \mathrm{~cm}$ \\
Weight & $70.7 \mathrm{Kg}$ \\
Waist circumference at navel height & $88.0 \mathrm{~cm}$ \\
Inside leg length to floor & $80.5 \mathrm{~cm}$ \\
Outside leg length iliac crest to floor & $102.0 \mathrm{~cm}$ \\
Thigh circumference at widest point & $51.8 \mathrm{~cm}$ \\
Knee circumference over patella & $38.5 \mathrm{~cm}$ \\
Calf circumference at widest point & $34.0 \mathrm{~cm}$ \\
Ankle circumference at medial malleolus level & $25.0 \mathrm{~cm}$ \\
Foot size & $26.5 \mathrm{~cm}$ \\
Walking speed & $0.59 \mathrm{~m} / \mathrm{s}$ \\
Cadence & $50 \mathrm{steps} / \mathrm{min}$ \\
\hline
\end{tabular}

Before the dynamic trials were performed, the participant executed knee flexion and hip rotation for a modified functional calibration of the knee joint center and axis of rotation and hip joint center, respectively [23]. In addition, a static trial with additional markers placed on anatomical landmarks to define the joints was recorded. A combination of the functional calibration and anatomical landmark coordinates was used to define coordinate systems of the segments. Joint angles were calculated based on the standards defined by the International Society of Biomechanics [24].

The participant performed several trials of walking at selfselected speed. We selected one sample trial where both force plates were hit with the left and right foot respectively. All kinematic and kinetic data plotted in Fig. 1 is represented from 0 to $100 \%$ of gait cycle $(0 \%=$ touch-down of heel, $100 \%=$ consecutive touch-down of the same heel).

The participant shows a reduced push-off and reduced ankle plantarflexion at the end of the stance phase which can be seen in Fig. 1 (ankle plantarflexion angle), where the torques are smaller and the ankle plantarflexion angle is reduced in the affected right side compared to the unaffected left side. Although the participant shows altered ankle kinematics, he does not present typical drop-foot kinematics. He maintains his affected ankle in more dorsiflexion at the end of stance and during swing compared to the unaffected side. This indicates that the dorsiflexor muscles are active. But they may be unable to perform the fast contraction needed to perform a fast dorsiflexion to prevent tripping. This assumption is supported by the reduced power at the ankle in the late stance phase.

At the knee, the flexion at the beginning of stance (at $10 \%$ of gait cycle) is clearly reduced for the affected side, which is in agreement with the reduced knee extension moment at this moment of time (Fig. 1). We can notice also a reduction of the power in the early stand phase, and an abnormal increase of power in the last stand phase. This is probably due to the different gait strategy adopted by the subject, which forces him to use more energy at the knee and hip joint and less at the ankle in that phase of the gait.

The hip presents a smaller difference between affected and unaffected leg. Most of the differences can be explained as a result of a change of the walking strategy. 

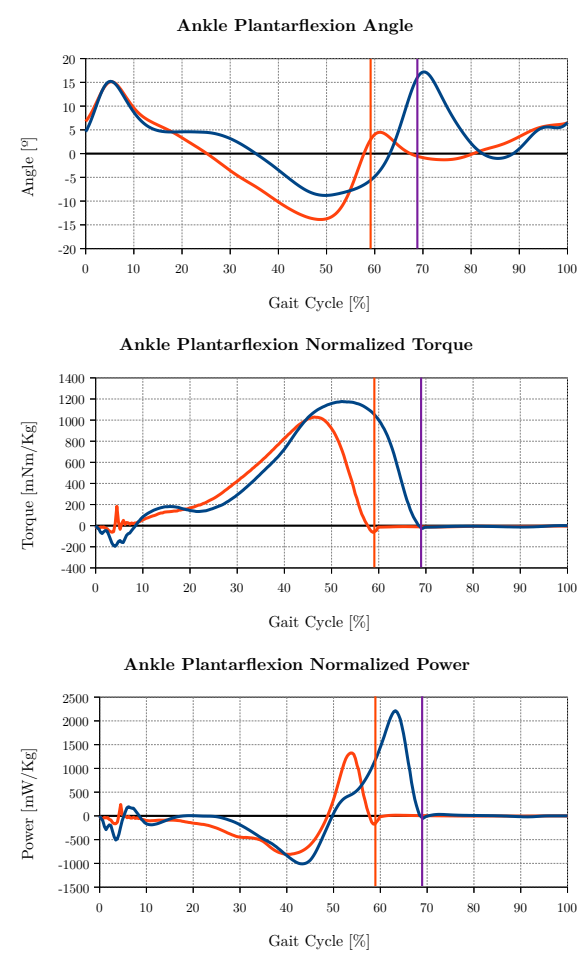

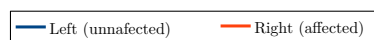

Knee Flexion Angle

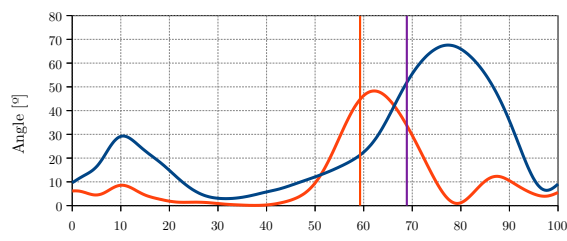

Gait Cycle [\%]
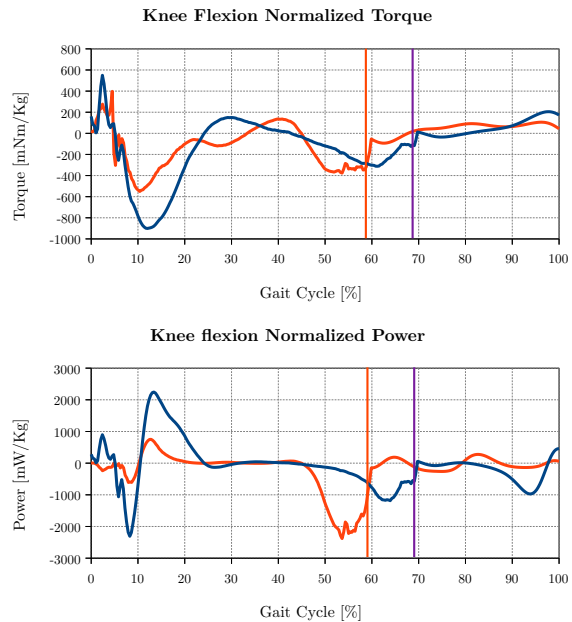

Hip Flexion Angle

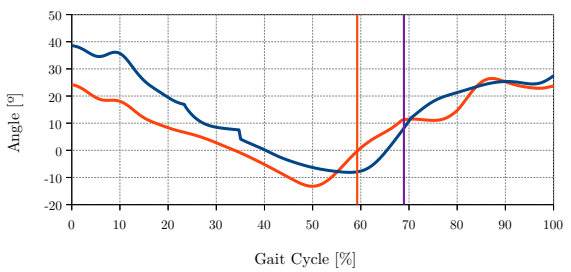

Hip Flexion Normalized Torque

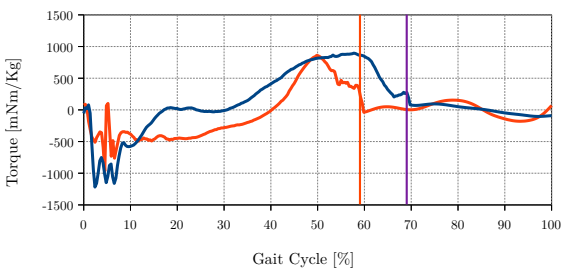

Hip Flexion Normalized Power

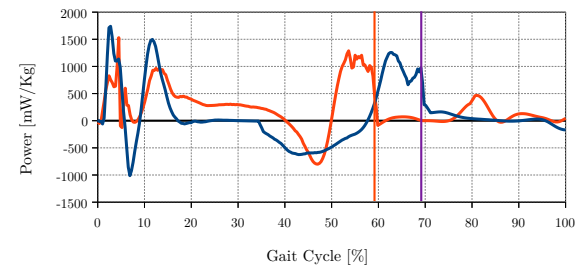

Fig. 1. Subject recorded gait data. The left leg (unaffected) is represented in blue and the right leg (affected) in red. All graphs are plotted from $0 \%$ to $100 \%$ of gait cycle $(0 \%=$ touch-down of heel, $100 \%=$ consecutive touch-down of the same heel). The toe-off moment is represented with blue and red vertical lines for the left and right legs respectively. The left column represents the ankle, the middle column the knee and the right column the hip. The top row represents the angle, the middle row the normalized torque and he bottom row the normalized power.

\section{A. Data pre-processing}

In order to apply the proposed method to this specific case we take the following assumption: Applying an assistive torque to the affected leg equal to the torque difference between the unaffected and the affected leg, the normal gait will be restored. Following this assumption, we generate a new data set consisting on the assistive torque (reference torque), and the motion of the unaffected leg. In order to do this, and to prepare the data for the subsequent optimization, we perform the following pre-processing steps:

- Phase matching. As shown in Fig. 1, the affected leg presents a shift in the toe-off moment with respect to the unaffected leg. In this first step, we adjust the time frame of the stand and swing phases to match both legs.

- Torque difference. Once the data from both legs is synchronized, the difference between the torque applied by the affected and unaffected leg is calculated. This difference should give us an approximation of the torque required to restore the walking gait on the affected leg.

- Filtering. This step is necessary to remove the noise present in the first points of the torque data, which can be noticed in Fig. 1.

- Cyclic filtering. The joint angle should be a cyclic movement. However, in a real scenario there could be some variation between gait cycles, which could lead to different joint angles at the begin and end of the data set. We apply a filter between the last and first points of the data set to ensure that the dataset is cyclic.

\section{B. Linear mechanism}

To implement an assistive wearable device on any of the above mentioned joints, we could use a mechanism to directly apply the desired torque through a rigid structure. However, if the wearable device is meant to be soft, the torque cannot be applied directly on the human joint, but using a linear transmission system such as a string or cord. In this case, to proceed with the mechanism optimization, we need to convert the joint torque to applied force, and joint angle to elongation. In order to do this, we will apply a simplification of the mechanism according to Fig. 2. As we can see in the figure, we can use a cord to pull from the back of the joint to assist the flexion of the joint, or to pull from the front to assist the extension of the joint.

The cord length and the lever arm in the flexion and extension mechanisms will change with the variation of the joint angle, according to equations $12-13$ and 14-15 respectively. The graphical representation of the cord length and lever arm for these mechanisms is depicted in Fig. 3.

$$
\begin{gathered}
L_{\text {flex }}=L^{\prime} \sqrt{2-2 \cos (\beta)} \\
R_{\text {flex }}=\frac{L^{\prime 2}}{L_{\text {flex }}} \sin \beta
\end{gathered}
$$



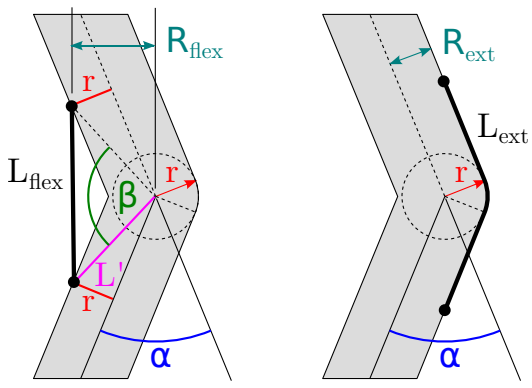

Fig. 2. Linear actuation system mounted on a knee joint. The actuation can assist the flexion (left scheme) or the extension (right scheme) of the joint depending on the configuration.

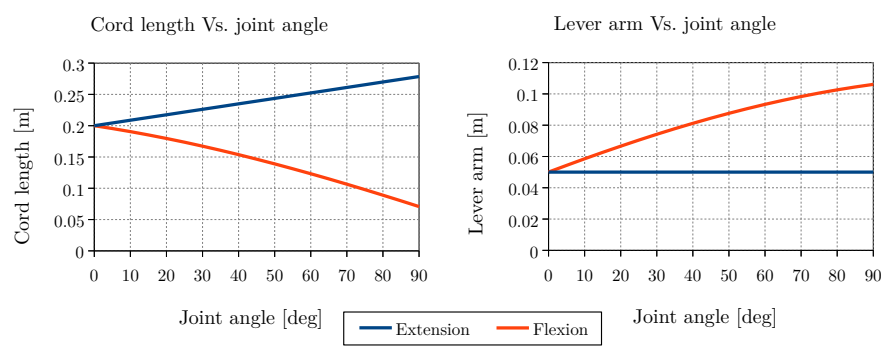

Fig. 3. Variation of the cord length and lever arm for the flexion and extension mechanisms, with $\mathrm{r}=0.05 \mathrm{~m}$ and $\mathrm{L}=0.2 \mathrm{~m}$

$$
\begin{gathered}
L_{e x t}=L+r \alpha \\
R_{e x t}=r
\end{gathered}
$$

where

$$
\begin{gathered}
L^{\prime}=\sqrt{L^{2} / 4+r^{2}} \\
\beta=\pi-\alpha-2 \cdot \operatorname{atan}\left(\frac{2 r}{L}\right)
\end{gathered}
$$

$\alpha$ is the joint angle, $L$ is the cord length in the neutral position $(\alpha=0)$ and $r$ is the joint radius.

In particular, the initial length and radius of the linear mechanism has been chosen according to the anthropometric values of the subject (see Table II). Table III contains the parameters used for the linear mechanism for each joint and configuration.

\section{Mechanism Design And Optimization}

Using the processed data, we can run the optimization algorithm based on the formulation described in section II-D

TABLE III

LINEAR MECHANISM

\begin{tabular}{llcc}
\hline Joint & Configuration & Radius [m] & Length [m] \\
\hline Ankle & Dorsiflexion & 0.06 & 0.30 \\
Ankle & Plantarflexion & 0.09 & 0.30 \\
Knee & Flexion/Extension & 0.07 & 0.60 \\
Hip & Flexion/Extension & 0.15 & 0.60 \\
\hline
\end{tabular}

TABLE IV

COMPONENT SET

\begin{tabular}{lccl}
\hline Component & Symbol & Parameter & Description \\
\hline Elastic cord & EC & $\begin{array}{c}k[\mathrm{~N} / \mathrm{m}] \\
x_{0}[\mathrm{~m}]\end{array}$ & $\begin{array}{l}\text { Stiffness } \\
\text { Offset }\end{array}$ \\
\hline Damper & DA & $c[\mathrm{Ns} / \mathrm{m}]$ & Damping coefficient \\
\hline Variable damper & $\mathrm{VD}$ & $c i[\mathrm{Ns} / \mathrm{m}]$ & $\begin{array}{l}\text { Damping coefficients } \\
i=1 \ldots 5\end{array}$ \\
\hline \multirow{2}{*}{ Clutch } & $\mathrm{CL}$ & $\begin{array}{c}T_{e}[\mathrm{~s}] \\
T_{d}[\mathrm{~s}]\end{array}$ & $\begin{array}{l}\text { Engage time } \\
\text { Disengage time }\end{array}$ \\
\hline
\end{tabular}

and let the system to test different combinations of basic components. For each mechanism, the system will calculate the optimal parameters, as well as the improvement in terms of energy. Table IV shows the components which have been made available to the system and their corresponding parameters and descriptions. Some of these components are easy to implement in a real application, for example the elastic cord can be simply a rubber band. However, the implementation of a variable damper or clutch on a soft structure is challenging. Hard components can be used (for example an electromagnetic or a magnetorheological clutch) in combination with a cable driven mechanism to transmit the assistive forces. This approach is however not the best solution due to several issues, such as weight and inertia. Further research on soft actuation technologies is being done for the practical implementation of the proposed components.

We have optimized the system in two different configurations for each joint: dorsiflexion and plantarflexion for the ankle, and flexion and extension for the knee and hip. To avoid excessive complexity of the mechanism, we limited the depth (number of parallel/series arrangements) of the mechanism to two, which means a maximum of four elements in any parallel/series combination. A more complex arrangement could probably grant better results, but at the expense of more weight and mechanical and control complexity. Table $\mathrm{V}$ depicts the best five mechanisms arrangements and their corresponding optimization error. The improvement of the mechanism is represented as a percentage of the residual error with respect to the reference error (the joint without exoskeleton), where the lower is the percentage the better is the performance. We can see how the combination of elastic cord and a variable damper is in most of the cases one of the best solutions. Table VI shows the best parameter set for each joint configuration.

For practical reasons, it is not always feasible to implement the arrangement proposed by the optimization algorithm. There are a number of implementation issues which need to be taken into account, such as maximum and minimum stiffness, maximum damping, damping variation, etc. The body attachments will have certain compliance, which has to be considered during the design of the system. For example, a very stiff mechanism could potentially transmit the required forces with a very small displacement. However, in a practical implementation, the body attachment will present some deformation, which could be of the same order of the deformation required by the mechanism. In a similar way, a variable damping system will have a minimum and a maximum damping coefficient, and the quotient between them 
TABLE V

TOP 5 SOLUTIONS PER MECHANISM

\begin{tabular}{lcl}
\hline Error [W] & Rel. err. & Description \\
\hline \multicolumn{2}{l}{ Ankle plantarflexion } & \\
\hline 8.105 & $100.00 \%$ & Reference \\
$\mathbf{4 . 6 4 3}$ & $\mathbf{5 7 . 2 9 \%}$ & Parallel VD and series EC-CL \\
4.657 & $57.46 \%$ & Parallel EC and series EC-CL \\
4.657 & $57.46 \%$ & Series EC-CL \\
4.657 & $57.46 \%$ & Parallel CL and series EC-CL \\
4.658 & $57.47 \%$ & Parallel series EC-CL and series EC-CL \\
\hline Ankle dorsiflexion & \\
\hline 8.105 & $100.00 \%$ & Reference \\
$\mathbf{6 . 1 8 2}$ & $\mathbf{7 6 . 2 7 \%}$ & Parallel series EC-CL and series EC-CL \\
6.385 & $78.78 \%$ & Parallel VD and series EC-CL \\
7.011 & $86.50 \%$ & Parallel VD and EC \\
7.011 & $86.50 \%$ & Variable DA \\
7.012 & $86.51 \%$ & Parallel VD and DA \\
\hline Knee extension & & \\
\hline 19.964 & $100.00 \%$ & Reference \\
$\mathbf{1 1 . 6 7 4}$ & $\mathbf{5 8 . 4 8 \%}$ & Parallel VD and series EC-CL \\
11.933 & $59.77 \%$ & Parallel series EC-CL and series EC-CL \\
12.597 & $63.10 \%$ & Series EC-CL \\
12.629 & $63.26 \%$ & Parallel EC and series EC-CL \\
12.987 & $65.05 \%$ & Parallel CL and series EC-CL \\
\hline Knee flexion & & \\
\hline 19.964 & $100.00 \%$ & Reference \\
$\mathbf{1 6 . 0 2 4}$ & $\mathbf{8 0 . 2 6 \%}$ & Parallel VD and series EC-CL \\
16.617 & $83.23 \%$ & Parallel EC and series EC-CL \\
16.618 & $83.24 \%$ & Series EC-CL \\
17.958 & $89.95 \%$ & Parallel series EC-CL and series EC-CL \\
18.442 & $92.38 \%$ & Parallel CL and series EC-CL \\
\hline Hip extension & & \\
\hline 21.769 & $100.00 \%$ & Reference \\
$\mathbf{7 . 4 2 0}$ & $\mathbf{3 4 . 0 9 \%}$ & Parallel series EC-CL and series EC-CL \\
8.639 & $39.68 \%$ & Parallel VD and series EC-CL \\
9.900 & $45.48 \%$ & Parallel DA and series EC-CL \\
9.930 & $45.62 \%$ & Parallel EC and series EC-CL \\
9.930 & $45.62 \%$ & Series EC-CL \\
\hline Hip flexion & & \\
\hline 21.769 & $100.00 \%$ & Reference \\
$\mathbf{7 . 5 3 2}$ & $\mathbf{3 4 . 6 0 \%}$ & Series EC-CL \\
7.532 & $34.60 \%$ & Parallel EC and series EC-CL \\
7.698 & $35.36 \%$ & Parallel series EC-CL and series EC-CL \\
9.462 & $43.47 \%$ & Parallel VD and series EC-CL \\
12.030 & $55.26 \%$ & Variable DA \\
\hline & &
\end{tabular}

TABLE VI

BEST MECHANISM PARAMETERS

\begin{tabular}{l|cccc}
\hline Joint & $k[\mathbf{N} / \mathbf{m}]$ & $c[\mathbf{N s} / \mathbf{m}]$ & $T_{e}[\mathbf{s}]$ & $T_{d}[\mathbf{s}]$ \\
\hline Ankle plantarflexion & 13873.00 & {$[0.32,288.56]$} & 0.10 & 0.82 \\
\hline Ankle dorsiflexion & 10462.10 & 1063.64 & 0.43 & 0.60 \\
& 7409.29 & 85.81 & 0.01 & 0.57 \\
\hline Knee extension & 16904.40 & {$[0.65,2645.44]$} & 0.00 & 0.64 \\
\hline Knee flexion & 19027.00 & {$[0.02,1509.61]$} & 0.02 & 0.14 \\
\hline Hip extension & 11674.80 & 865.70 & 0.18 & 0.68 \\
& 11876.60 & 817.25 & 0.44 & 0.71 \\
\hline Hip flexion & 2588.74 & 71.41 & 0.15 & 0.65 \\
\hline
\end{tabular}

will be limited by the technology. The proposed mechanism could be as the one shown in Fig. 5. In the next subsections we analyze more in detail the best mechanisms (written in bold in Table V) for the ankle, knee and hip actuation.

\section{A. Ankle design}

On the left column of Fig. 4, we can see the behavior of the proposed mechanism assisting the ankle plantarflexion (top) and ankle dorsiflexion (bottom). There are some evident differences between both systems, as it is also highlighted in
TABLE VII

MECHANISM OPTIMIZATION RESULTS

\begin{tabular}{|c|c|c|c|}
\hline & w/o exo & with exo & Improvement \\
\hline \multicolumn{4}{|c|}{ Ankle plantarflexion (Parallel VD and series EC-CL) } \\
\hline Peak force $[N]$ & 331.7 & 185.5 & $-44.1 \%$ \\
\hline Peak power $[\mathrm{W}]$ & 69.2 & 44.5 & $-35.7 \%$ \\
\hline Energy $[\mathrm{J}]$ & 9.86 & 5.65 & $-42.8 \%$ \\
\hline \multicolumn{4}{|c|}{ Ankle dorsiflexion (Parallel series EC-CL and series EC-CL) } \\
\hline Peak force $[\mathrm{N}]$ & 221.1 & 221.1 & $-0.0 \%$ \\
\hline Peak power $[\mathrm{W}]$ & 69.2 & 69.2 & $-0.0 \%$ \\
\hline Energy $[J]$ & 9.86 & 7.52 & $-23.7 \%$ \\
\hline \multicolumn{4}{|c|}{ Knee extension (Parallel VD and series EC-CL) } \\
\hline Peak force $[\mathrm{N}]$ & 353.2 & 257.0 & $-27.2 \%$ \\
\hline Peak power $[\mathrm{W}]$ & 93.5 & 93.7 & $+0.24 \%$ \\
\hline Energy $[J]$ & 24.27 & 14.18 & $-41.6 \%$ \\
\hline \multicolumn{4}{|c|}{ Knee flexion (Parallel VD and series EC-CL) } \\
\hline Peak force $[N]$ & 237.0 & 230.7 & $-2.7 \%$ \\
\hline Peak power $[\mathrm{W}]$ & 93.5 & 94.1 & $+0.72 \%$ \\
\hline Energy $[J]$ & 24.27 & 19.48 & $-19.8 \%$ \\
\hline \multicolumn{4}{|c|}{ Hip extension (Parallel series EC-CL and series EC-CL) } \\
\hline Peak force $[N]$ & 329.1 & 148.7 & $-54.8 \%$ \\
\hline Peak power $[\mathrm{W}]$ & 57.8 & 52.2 & $-9.7 \%$ \\
\hline Energy $[J]$ & 26.49 & 9.02 & $-65.9 \%$ \\
\hline \multicolumn{4}{|c|}{ Hip flexion (Series EC-CL) } \\
\hline Peak force $[N]$ & 350.3 & 156.9 & $-55.2 \%$ \\
\hline Peak power $[\mathrm{W}]$ & 57.8 & 47.8 & $-17.4 \%$ \\
\hline Energy $[J]$ & 26.49 & 9.16 & $-65.4 \%$ \\
\hline
\end{tabular}

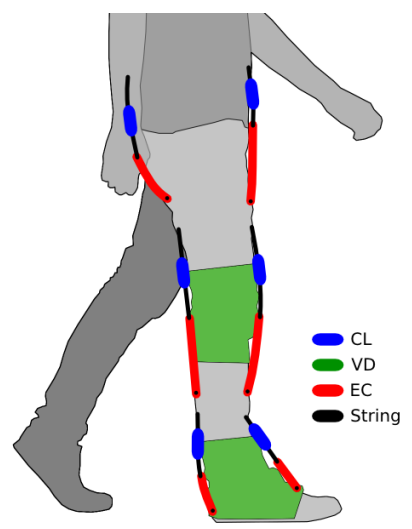

Fig. 5. Possible implementation of the proposed mechanisms. Note that the Variable Damper (VD) of the knee and ankle joints could be implemented around the joint instead of with a linear mechanism.

Table VII. The plantarflexion mechanism is able to reduce by $42.8 \%$ the energy of the joint, as well as the force and power peaks ( $44.1 \%$ and $35.7 \%$ respectively). The required force gets closer to zero, and the power is almost flat with just a few remaining peaks in the early stand phase and at the end of the push-off. The dorsiflexion mechanism fails however to reduce the force and power peaks, but it reduces the required energy by a $23.7 \%$.

\section{B. Knee design}

In the case of the knee joint, the extension mechanism performs better than the flexion mechanism (see central column of Fig. 4 and Table VII). In both cases there is an important reduction of the energy ( $41.6 \%$ and $19.8 \%$ respectively), but only the extension mechanism is able to provide a representative reduction of the peak force $(27.2 \%)$. Seeing in detail the power of the extension mechanism, we can see how the 

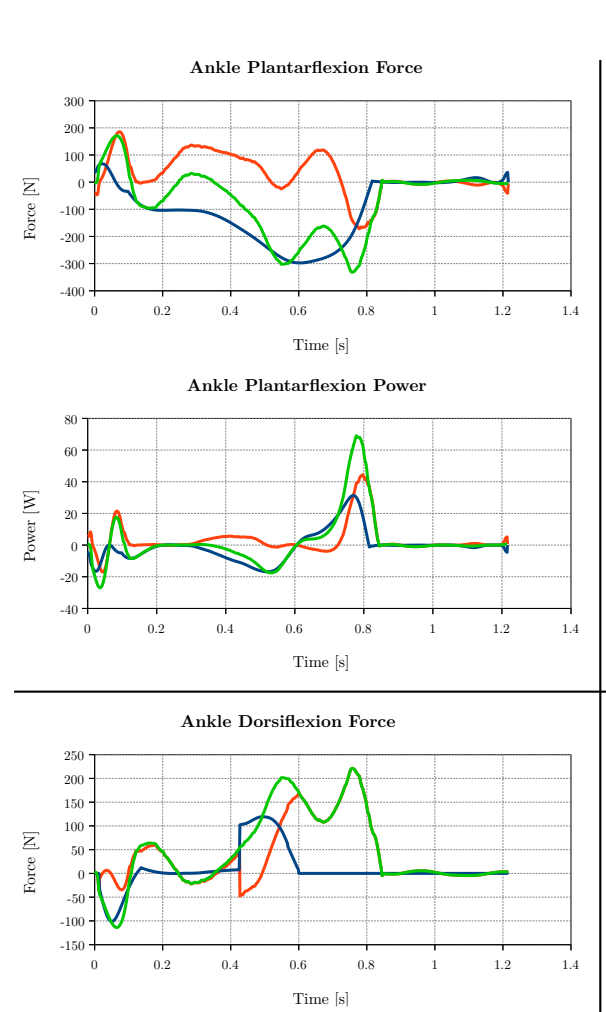

Ankle Dorsiflexion Power

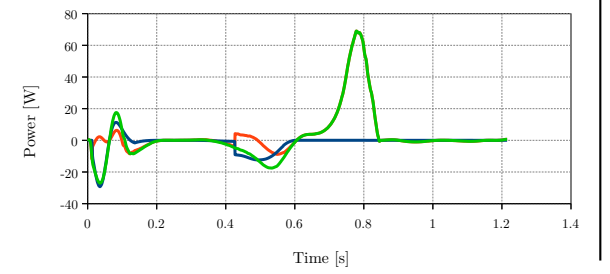

一 Ref -Exo - Req

Knee Extension Force

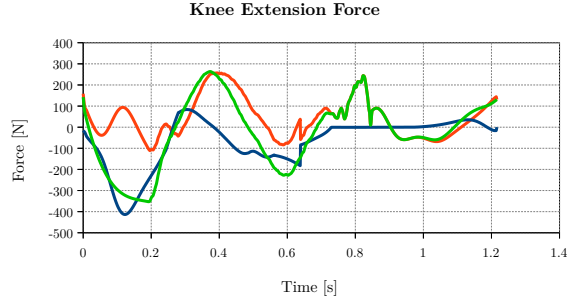

Knee Extension Power

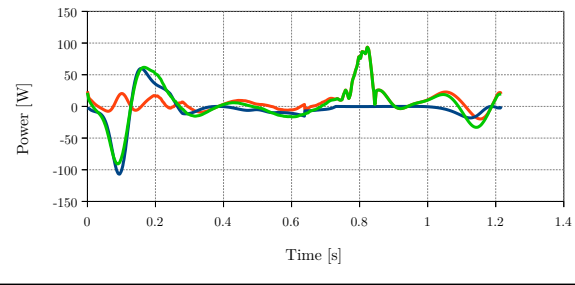

Knee Flexion Force

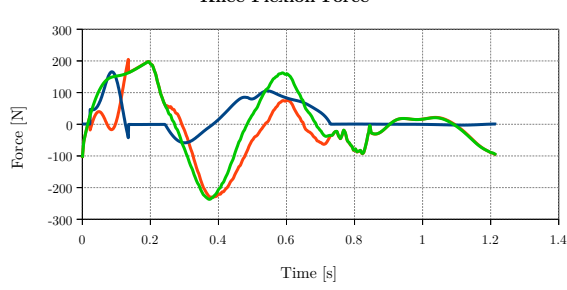

Knee Flexion Power

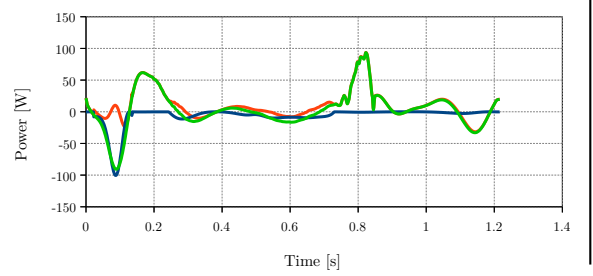

Hip Extension Force

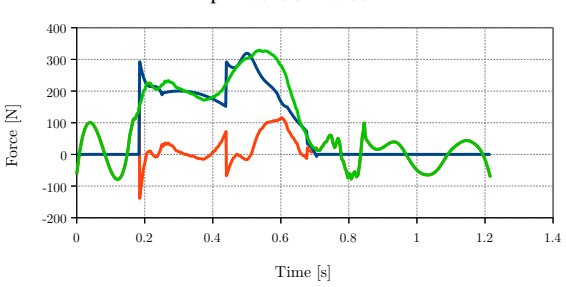

Hip Extension Power

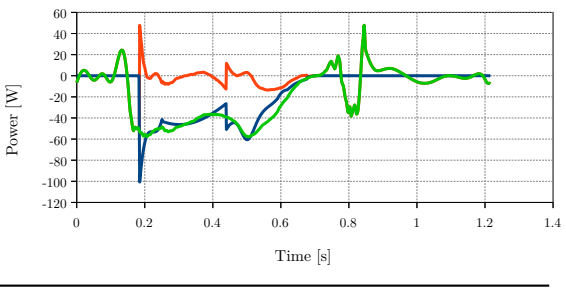

Hip Flexion Force

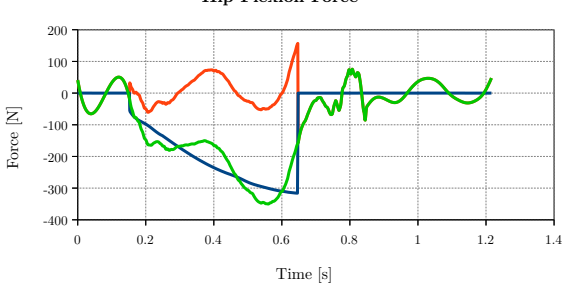

Hip Flexion Power

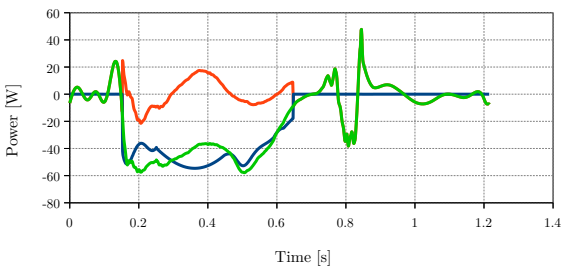

Fig. 4. Comparison between the force (top graph) and power (bottom graph) during the plantarflexion and dorsiflexion of the ankle (left column), extension and flexion of the knee (middle column) and extension and flexion of the hip (right column). The reference force (Ref) is reduced by the action of the exoskeleton (Exo). The difference between them (Req) is calculated following the equation 1.

system is able to reduce close to zero the power requirements during most of the gait phase. However, the power peak at the end of the stand phase remains practically unmodified.

\section{Hip design}

The proposed hip designs, for flexion and extension, work in a very similar way. On the right column of Fig. 4, we can see how the force in the extension and flexion cases roughly follow the reference force profile. Note that the optimized flexion mechanism is composed by an elastic cord and a clutch in series configuration, and during the gait motion there is only one 'on' and one 'off' phase (with transitions at $0.15 \mathrm{~s}$ and $0.65 \mathrm{~s}$ ). However, in the extension mechanism the best solution combines in parallel two of these mechanisms, having four different phases (with transitions at $0.18 \mathrm{~s}, 0.44 \mathrm{~s}, 0.68 \mathrm{~s}, 0.71$ s). Both mechanisms are able to reduce a great part of the system energy $(65 \%)$, and force and power peaks (see Table VII).

\section{Conclusions}

The design of the mechanism of a wearable robot is highly dependent on the target task. The combination of passive elements (springs, elastic cords, dampers), and quasi-passive elements (variable dampers, clutches) allows to improve the energy efficiency of the system. However, the arrangement of these elements is not trivial. In this paper we presented a simplified formulation which allows to perform an optimization of an arbitrary arrangement of mechanical components, and we used this strategy for the optimization of an ankle, knee and hip actuation system using real subject data.

The results show how the proposed mechanisms have the potential to assist the motion of a lower limb, in the analyzed case reducing the differences between the affected and unaffected legs during walking. In the best cases with a reduction of the energy requirements of $65 \%$. The actuator arrangements proposed by the optimization algorithms are the base for the actuation system of the XoSoft exoskeleton, which are currently under development. The practical implementation 
of such mechanisms might differ from the ideal one used in simulation.

It is also possible to use the described method to analyze a different set of tasks, such as stand-to-sit, sit-to-stand, stairs negotiation, walking and turning, and so on. As a first approach, we selected the straight line walking since it is the most relevant task in daily living with the highest potential impact for the subjects. In the same way, the proposed method could be applied to a pool of subjects with similar pathologies to extract a common mechanism arrangement, but with specific parameters for each subject.

The method proposed in this paper, could be improved with a more accurate modelization of the different elements, also including experimental characterization of real implementations. For example, including non linearities and hysteresis in the elastic cord stiffness, friction of the cable mechanism, transition between on/off phases of the clutch, etc. In particular, the ideal clutch used in the simulations produces high frequency components in the force, which a real system would not present. In future steps, the actual performance of the real system will be evaluated and compared with the simulation results.

Another improvement of the system is the possibility to perform an optimization across more than one single joint. The optimization of a mechanism actuating simultaneously two or more joints is possible using the same system described in this paper, but using as input data the combined information from all the joints involved. This system has been already successfully tested and it will be used in the next iterations of the design of the system.

One limitation of the proposed method is that it is not able to simulate and optimize two mechanisms actuating simultaneously in a single joint. For example, the combination of two mechanisms working in the flexion and extension directions. In future developments we plan to include the geometry of the mechanism within the simulation and optimization functions, which would allow not only to simulate opposed mechanisms, but also to optimize the geometric description of the mechanism, such as joint radius and lever arm.

It is important to note that the proposed method could be applied to other mechanism optimization problems, and not only for wearable robots, such as humanoid or quadruped robots.

\section{REFERENCES}

[1] J. L. Pons et al., "Wearable robots: biomechatronic exoskeletons," 2008.

[2] K. Low, "Robot-assisted gait rehabilitation: From exoskeletons to gait systems," in Defense Science Research Conference and Expo (DSR), 2011. IEEE, 2011, pp. 1-10.

[3] A. Zoss, H. Kazerooni, and A. Chu, "On the biomechanical design of the berkeley lower extremity exoskeleton (bleex)," IEEE/ASME Transactions on Mechatronics, vol. 11, no. 2, pp. 128-138, April 2006.

[4] M. Wehner, B. Quinlivan, P. M. Aubin, E. Martinez-Villalpando, M. Baumann, L. Stirling, K. Holt, R. Wood, and C. Walsh, "A lightweight soft exosuit for gait assistance," in Robotics and Automation (ICRA), 2013 IEEE International Conference on. IEEE, 2013, pp. $3362-3369$.

[5] S. Toxiri, J. Ortiz, J. Masood, J. Fernández, L. A. Mateos, and D. G. Caldwell, "A wearable device for reducing spinal loads during lifting tasks: Biomechanics and design concepts," in Robotics and Biomimetics (ROBIO), 2015 IEEE International Conference on. IEEE, 2015, pp. 2295-2300.
[6] J. Ortiz, E. Rocon, V. Power, A. de Eyto, L. OSullivan, M. Wirz, C. Bauer, S. Schülein, K. S. Stadler, B. Mazzolai, W. B. Teeuw, C. Baten, C. Nikamp, J. Buurke, F. Thorsteinsson, and J. Müller, "Xosoft-a vision for a soft modular lower limb exoskeleton," in Wearable Robotics: Challenges and Trends. Springer International Publishing, 2017, pp. $83-88$.

[7] V. Power, L. O'Sullivan, A. de Eyto, S. Schülein, C. Nikamp, C. Bauer, J. Müller, and J. Ortiz, "Exploring user requirements for a lower body soft exoskeleton to assist mobility," in Proceedings of the 9th ACM International Conference on PErvasive Technologies Related to Assistive Environments. ACM, 2016, p. 69.

[8] K. Endo, D. Paluska, and H. Herr, "A quasi-passive model of human leg function in level-ground walking," in 2006 IEEE/RSJ International Conference on Intelligent Robots and Systems. IEEE, 2006, pp. 49354939.

[9] C. J. Walsh, K. Endo, and H. Herr, "A quasi-passive leg exoskeleton for load-carrying augmentation," International Journal of Humanoid Robotics, vol. 4, no. 03, pp. 487-506, 2007.

[10] P. Cherelle, V. Grosu, P. Beyl, A. Mathys, R. Van Ham, M. Van Damme, B. Vanderborght, and D. Lefeber, "The maccepa actuation system as torque actuator in the gait rehabilitation robot altacro," in Biomedical Robotics and Biomechatronics (BioRob), 2010 3rd IEEE RAS and EMBS International Conference on. IEEE, 2010, pp. 27-32.

[11] M. Moltedo, T. Bacek, K. Junius, B. Vanderborght, and D. Lefeber, "Mechanical design of a lightweight compliant and adaptable active ankle foot orthosis," in Biomedical Robotics and Biomechatronics (BioRob), 2016 6th IEEE International Conference on. IEEE, 2016, pp. 12241229.

[12] T. Bacek, M. Moltedo, J. Gonzalez-Vargas, G. A. Prieto, M. SanchezVillamañan, J. Moreno, and D. Lefeber, "The new generation of compliant actuators for use in controllable bio-inspired wearable robots," in Wearable Robotics: Challenges and Trends. Springer, 2017, pp. 255-259.

[13] A. Cullell, J. C. Moreno, E. Rocon, A. Forner-Cordero, and J. L. Pons, "Biologically based design of an actuator system for a knee-ankle-foot orthosis," Mechanism and Machine Theory, vol. 44, no. 4, pp. 860-872, 2009.

[14] J. Masood, J. Ortiz, J. Fernández, L. A. Mateos, and D. G. Caldwell, "Mechanical design and analysis of light weight hip joint parallel elastic actuator for industrial exoskeleton," in Biomedical Robotics and Biomechatronics (BioRob), 2016 6th IEEE International Conference on. IEEE, 2016, pp. 631-636.

[15] K. W. Hollander, R. Ilg, T. G. Sugar, and D. Herring, "An efficient robotic tendon for gait assistance," Journal of biomechanical engineering, vol. 128, no. 5, pp. 788-791, 2006.

[16] E. J. Rouse, L. M. Mooney, and H. M. Herr, "Clutchable series-elastic actuator: Implications for prosthetic knee design," The International Journal of Robotics Research, vol. 33, no. 13, pp. 1611-1625, 2014.

[17] M. Xiloyannis, L. Cappello, K. D. Binh, C. W. Antuvan, and L. Masia, "Preliminary design and control of a soft exosuit for assisting elbow movements and hand grasping in activities of daily living," Journal of Rehabilitation and Assistive Technologies Engineering, vol. 4, p. $2055668316680315,2017$.

[18] W. Roozing, Z. Li, D. G. Caldwell, and N. G. Tsagarakis, "Design optimisation and control of compliant actuation arrangements in articulated robots for improved energy efficiency," IEEE Robotics and Automation Letters, vol. 1, no. 2, pp. 1110-1117, 2016.

[19] O. Sigmund, "On the design of compliant mechanisms using topology optimization," Journal of Structural Mechanics, vol. 25, no. 4, pp. $493-$ $524,1997$.

[20] J. Hetrick and S. Kota, "An energy formulation for parametric size and shape optimization of compliant mechanisms," Journal of Mechanical Design, vol. 121, no. 2, pp. 229-234, 1999.

[21] T. E. Bruns and D. A. Tortorelli, "Topology optimization of non-linear elastic structures and compliant mechanisms," Computer Methods in Applied Mechanics and Engineering, vol. 190, no. 2627, pp. 3443-3459, 2001.

[22] J. A. Nelder and R. Mead, "A simplex method for function minimization," The computer journal, vol. 7, no. 4, pp. 308-313, 1965.

[23] R. List, T. Gülay, M. Stoop, and S. Lorenzetti, "Kinematics of the trunk and the lower extremities during restricted and unrestricted squats," The Journal of Strength \& Conditioning Research, vol. 27, no. 6, pp. 1529 1538, 2013.

[24] E. S. Grood and W. J. Suntay, "A joint coordinate system for the clinical description of three-dimensional motions: application to the knee," Journal of biomechanical engineering, vol. 105, no. 2, pp. 136144, 1983. 